Cite this: Phys. Chem. Chem. Phys., 2013, 15, 21016

Received 16th July 2013,

Accepted 4th October 2013

DOI: $10.1039 / c 3$ cp52976b

www.rsc.org/pccp

\title{
Enhanced hydrogen sensing properties of graphene by introducing a mono-atom-vacancy
}

\author{
Q. G. Jiang, ${ }^{a b}$ Z. M. Ao, ${ }^{* b c}$ W. T. Zheng, ${ }^{a}$ S. Li ${ }^{b}$ and Q. Jiang*a
}

\begin{abstract}
To facilitate the dissociative adsorption of $\mathrm{H}_{2}$ molecules on pristine graphene, the addition of a monoatom-vacancy to graphene is proposed. This leads to reduction of the dissociative energy barrier for a $\mathrm{H}_{2}$ molecule on graphene from 3.097 to $0.805 \mathrm{eV}$ for the first $\mathrm{H}_{2}$ and $0.869 \mathrm{eV}$ for the second, according to first principles calculations. As a result, two $\mathrm{H}_{2}$ molecules can be easily dissociatively adsorbed on this defected graphene at room temperature. The electronic structure and conductivity of the graphene change significantly after $\mathrm{H}_{2}$ adsorption. In addition, the related dissociative adsorption phase diagrams under different temperatures and partial pressures show that this dissociative adsorption at room temperature is very sensitive $\left(10^{-35} \mathrm{~mol} \mathrm{~L}^{-1}\right)$. Therefore, this defected graphene is promising for ultra-sensitive room temperature hydrogen sensing.
\end{abstract}

\section{Introduction}

Hydrogen has attracted great interest because it is a clean and renewable energy source. ${ }^{1}$ Hydrogen is widely present in nuclear reactors, coal mines and semiconductor manufacturing, etc. ${ }^{2}$ Because hydrogen is highly flammable and explosive with a volume concentration of more than $4 \%$, developing highly sensitive hydrogen sensors is important. Commercially, hydrogen sensors are mainly composed of metal oxide (e.g., $\mathrm{SnO}_{2}$ ) films based on the chemresistive mechanism. ${ }^{3-7}$ However, to obtain satisfactory results by using metal oxide sensors a temperature over $400{ }^{\circ} \mathrm{C}$ is usually required, ${ }^{8}$ which is energy intensive and may trigger an explosion. Therefore, it is desirable to develop new types of hydrogen sensors which operate at room temperature.

Although graphene has definite advantages over other gas sensing materials, ${ }^{9}$ due to its excellent electronic ${ }^{10}$ and mechanical properties, ${ }^{11}$ the interaction between pristine graphene and $\mathrm{H}_{2}$ molecules is weak due to the inert nature of graphene. ${ }^{12}$ It is known that when graphene is synthesized using chemical techniques, such as chemical vapor deposition (CVD) ${ }^{13}$ and aqueous dispersion methods, ${ }^{14}$ vacancies could be present. Vacancies can also be introduced into graphene using the ion irradiation technique. ${ }^{15,16}$ These vacancies can promote

\footnotetext{
${ }^{a}$ Key Laboratory of Automobile Materials (Jilin University), Ministry of Education, and School of Materials Science and Engineering, Jilin University, Changchun 130022, China. E-mail: jiangq@jlu.edu.cn

${ }^{b}$ School of Materials Science and Engineering, The University of New South Wales, Sydney, NSW 2052, Australia. E-mail: zhimin.ao@uts.edu.au

${ }^{c}$ Centre for Clean Energy Technology, School of Chemistry and Forensic Science, Faculty of Science, University of Technology, Sydney, PO Box 123, Broadway,

Sydney, NSW 2007, Australia
}

the reactivity of graphene and facilitate the dissociative adsorption of small molecules on graphene. ${ }^{17-19}$ Thus, we expect that $\mathrm{H}_{2}$ molecules could be dissociatively adsorbed on vacancies in the graphene and this adsorption may change the electronic properties of the graphene.

In this paper, we will consider the dissociative adsorption of $\mathrm{H}_{2}$ molecules on graphene with mono-atom-vacancies by using density functional theory (DFT) calculations. It is found that the defected graphene results in an evident drop of the dissociative energy barrier of $\mathrm{H}_{2}$. This adsorption evidently influences the conductivity of the defected graphene. In addition, the phase diagram of $\mathrm{H}_{2}$ dissociative adsorption on graphene under different temperatures and partial pressures is established based on DFT results and thermodynamic parameters, which confirm the sensitivity of hydrogen dissociative adsorption and the sensitivity of the graphene for hydrogen detection.

\section{Calculation methods and thermodynamic model}

All calculations are implemented by Dmol ${ }^{3}$ code. ${ }^{20}$ The generalized gradient approximation (GGA) with a Perdew-Burke-Ernzerhof (PBE) functional is employed to describe the exchange and correlation effects. ${ }^{21}$ Double numerical plus polarization (DNP) is employed as the basis set. A convergence tolerance of energy of $10^{-5}$ Hartree is taken $(1$ Hartree $=27.21 \mathrm{eV})$, and the maximal allowed force and displacement are 0.002 Hartree $\AA^{-1}$ and $0.005 \AA$, respectively. It was reported that the selection of the exchange-correlation functional has an evident effect on the resulting adsorption energies. However, the effect on the 
calculated energy barriers is much smaller. ${ }^{22}$ To investigate the minimum energy pathway for dissociative adsorption of a $\mathrm{H}_{2}$ molecule on graphene, the linear synchronous transit/quadratic synchronous transit (LST/QST) ${ }^{23}$ and nudged elastic band $(\mathrm{NEB})^{24}$ tools in the $\mathrm{Dmol}^{3}$ module are used, which have been well validated for determining the structure of the transition state and the minimum energy pathway. In the simulation, three-dimensional periodic boundary conditions are taken. The simulation cell consists of a $4 \times 4$ graphene supercell with a vacuum width of $20 \AA$ above the graphene layer to minimize the interlayer interaction. The $k$-point is set to $5 \times 5 \times 1$ and all atoms are allowed to relax. The DFT $+\mathrm{D}$ method within the Grimme scheme ${ }^{25}$ is used in all calculations to consider the van der Waals forces.

For a $\mathrm{H}_{2}$ molecule adsorbed on graphene before and after the dissociative adsorption, the adsorption energy $E_{\mathrm{ad}}$ is determined by,

$$
E_{\mathrm{ad}}=E_{\mathrm{H}_{2} / \text { graphene }}-\left(E_{\text {graphene }}+E_{\mathrm{H}_{2}}\right)
$$

where $E_{\mathrm{H}_{2}}$ /graphene, $E_{\text {graphene }}$ and $E_{\mathrm{H}_{2}}$ are the total energies of the $\mathrm{H}_{2}$ /graphene system, the isolated graphene and a $\mathrm{H}_{2}$ molecule in the supercell with the same lattice of graphene, respectively.

In actual applications, the temperature $(T)$ and pressure $(P)$ should be considered. Therefore, the Gibbs free energy $G(T, P)$ of the system was calculated as a function of $T$ and $P$. The change in Gibbs free energy for the dissociative adsorption of a $\mathrm{H}_{2}$ molecule on graphene is taken as,

$\Delta G(T, P)=G_{\mathrm{H}_{2} / \text { graphene }}(T, P)-G_{\text {graphene }}(T, P)-G_{\mathrm{H}_{2}}(T, P)$

The determined DFT total energy $E$ is related to the volume $V$ of the unit cell at $0 \mathrm{~K}$, corresponding to the Helmholtz free energy at $0 \mathrm{~K}$ without zero-point vibrations. Therefore, the Gibbs free energy $G(T, P)$ in eqn (2) can be determined by, ${ }^{26}$

$$
G(T, P)=E+F^{\mathrm{vib}}(T)+P V
$$

where $F^{\mathrm{vib}}(T)$ denotes the vibrational free energy. Because $G(T, P)$ of solid phases changes little with $P$, the $P V$ term in eqn (3) is neglected. ${ }^{26}$ Thus, $G_{\text {graphene }}(T, P)=E_{\text {graphene }}+F_{\text {graphene }}^{\text {vib }}(T)$ and $G_{\mathrm{H}_{2} / \text { graphene }}(T, P)=E_{\mathrm{H}_{2} / \text { graphene }}+F_{\mathrm{H}_{2} / \text { graphene }}^{\text {vib }}(T) . F^{\text {vib }}(T)$ within the harmonic approximation for $n$ fundamental modes (with a frequency of $\omega_{i}$ ) of the system is expressed as, ${ }^{27}$

$$
\begin{aligned}
F^{\mathrm{vib}}(T) & =\sum_{i=1}^{n} F^{\mathrm{vib}}\left(T, \omega_{i}\right) \\
& =\sum_{i=1}^{n} \frac{1}{2} \hbar \omega_{i}+K_{\mathrm{B}} T \ln \left[1-\exp \left(\frac{-\hbar \omega_{i}}{K_{\mathrm{B}} T}\right)\right] .
\end{aligned}
$$

$F^{\mathrm{vib}}(T)$ is thus determined by phonon calculations with CASTEP code ${ }^{28}$ where the norm-conserving pseudopotentials, the GGA-PBE functional, an energy cutoff of $340 \mathrm{eV}$ and $5 \times 5 \times 1$ $k$-point meshes are used.

The Gibbs free energy of the gas phase will be strongly affected by $T$ and $P$, and can be taken as, ${ }^{29}$

$$
\begin{aligned}
G_{\mathrm{H}_{2}}(T, P)= & E_{\mathrm{H}_{2}}+\Delta \mathrm{H}_{\mathrm{H}_{2}}\left(T, P^{0}\right)-T \Delta S_{\mathrm{H}_{2}} \\
& +K_{\mathrm{B}} T \ln \left(P / P^{0}\right)+F_{\mathrm{H}_{2}}^{\mathrm{vib}}(T)
\end{aligned}
$$

where the first term is the DFT result, the second and third terms are the contribution of $\mathrm{H}_{2}$ enthalpy and entropy under atmospheric pressure $\left(P^{0}=1 \mathrm{~atm}\right)$, respectively, which can be obtained from a thermodynamic database. ${ }^{30}$ The fourth term is a pressure dependent contribution (where $K_{\mathrm{B}}$ is the Boltzmann constant). In total, $\Delta G(T, P)$ in eqn (2) is further expressed as,

$$
\begin{aligned}
\Delta G(T, P)= & E_{\mathrm{H}_{2} / \text { graphene }}+F_{\mathrm{H}_{2} / \text { graphene }}^{\mathrm{vib}}(T)-E_{\text {graphene }} \\
& -F_{\text {graphene }}^{\mathrm{vib}}(T)-E_{\mathrm{H}_{2}}-\Delta H_{\mathrm{H}_{2}}\left(T, P^{0}\right) \\
& +T \Delta S_{\mathrm{H}_{2}}-K_{\mathrm{B}} T \ln \left(P / P^{0}\right)-F_{\mathrm{H}_{2}}^{\mathrm{vib}}(T)
\end{aligned}
$$

\section{Results and discussion}

We firstly consider the geometric structures of the pristine graphene and graphene with a mono-atom-vacancy as shown in Fig. 1, the corresponding vacancy concentration is $1 / 32$. Three $\mathrm{sp}^{2}$ dangling bonds ( $\sigma$ bonds) are present near the vacancy and point towards the center of the vacancy. In addition, one delocalized $\pi$ dangling bond is perpendicular to the graphene surface. The vacancy undergoes a Jahn-Teller distortion upon relaxation (see Fig. 1b), where two of the nearest $\mathrm{C}$ atoms to the vacancy site form a weak covalent bond with bond length $2.129 \AA$, consistent with the reported value of $2.1 \AA^{17}$ This distortion results in a pentagon-like structure, with the atom displaced 0.20 A out of the surface plane, which corresponds to the literature datum of $0.18 \AA \AA^{17}$ The ground state of the vacancy has a magnetic moment of $1.006 \mu_{\mathrm{B}}$ (see Fig. 1b), which is consistent with the reported value of $1.04 \mu_{\mathrm{B}}$ and experimental observation. ${ }^{17,31}$ To further understand the electronic structure of the defective graphene, the partial density of states (PDOS) of the $\mathrm{C}_{1}$ and $\mathrm{C}_{2}$ atoms are analyzed and shown in Fig. 1c and d, respectively.

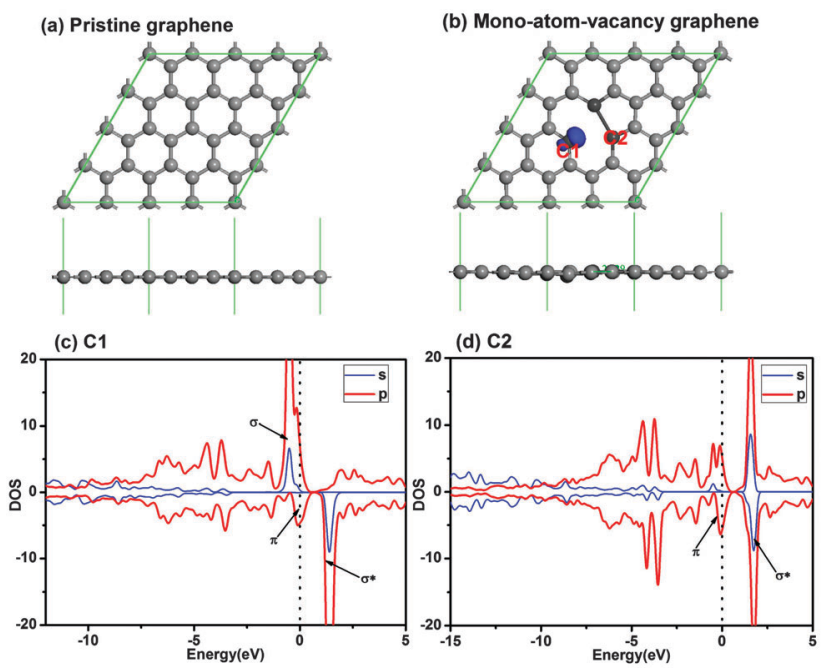

Fig. 1 The favorable configurations of pristine graphene (a), and graphene with a mono-atom-vacancy (b). The spin density of the vacancy is also shown in blue. $C_{1}$ and $C_{2}$ represent different carbon positions. The grey and black atoms are saturated and unsaturated $C_{1}$, respectively. The PDOS of the $C_{1}$ and $C_{2}$ atoms are shown in (c) and (d), respectively. The blue and red curves are the PDOS of the $s$ orbital and $p$ orbitals of the $C$ atoms, respectively. 
The spin state here is mainly contributed by the half filled $\sigma$ orbital of the $\mathrm{C}_{1}$ atom (see Fig. 1c). The $\mathrm{C}_{2}$ atom forms a weak bond with the neighboring $\mathrm{C}$ atom and builds a fully empty $\sigma^{*}$ anti-bond (see Fig. 1d) without the spin state. The half filled $\pi$ bond is responsible for the p-type doping, but not for the spin state of the graphene. Therefore, formation of the pentagon saturates two of three $\mathrm{sp}^{2}$ bonds, but the remaining unsaturated $\sigma$ bond of the $\mathrm{C}_{1}$ atom is reactive and is believed to induce the dissociative adsorption of small molecules.

For the molecular adsorption of a $\mathrm{H}_{2}$ molecule on pristine graphene, based on the DFT $+\mathrm{D}$ calculations and eqn (1), the favorable adsorption site of the $\mathrm{H}_{2}$ molecule is at the hollow site with the $\mathrm{H}-\mathrm{H}$ bond parallel to the graphene surface, which is consistent with ref. 12 and shown as the initial structure, IS, in the inset of Fig. 2a. Note that it has been reported that the $\mathrm{DFT}+\mathrm{D}$ method tends to over-emphasize the pair-wise interaction energy between atoms and gives the wrong binding site for Ag atoms on graphite. ${ }^{32,33}$ To check the correctness of our result, the literature results using vdW-DF and vdW-WF methods are compared, ${ }^{34}$ which give the same favorable adsorption position for the $\mathrm{H}_{2}$ molecule on graphene as our study. For the $\mathrm{H}_{2}$ dissociative adsorption on graphene, the most favorable configuration is with two $\mathrm{H}$ atoms chemisorbed on the two face-to-face $\mathrm{C}$ atoms in the six-member ring, as shown as the final structure, FS, in Fig. 2a. LST/QST and NEB calculations indicate that this reaction is endothermic with a high energy barrier, $E_{\mathrm{bar}}$, of $3.097 \mathrm{eV}$ (the energy difference between the transition state, TS, and the initial structure, IS), which is a little smaller than the reported result of $3.3 \mathrm{eV}{ }^{12}$ Therefore, the dissociative adsorption of $\mathrm{H}_{2}$ on pristine graphene is difficult to achieve.

The obtained adsorption energy $E_{\text {ad_Is }}$, based on eqn (1), for graphene with a mono-atom-vacancy is listed in Table 1. The preferred structure of a $\mathrm{H}_{2}$ molecule when adsorbed in this system is shown in Fig. 2b, which is taken as the IS in the subsequent transition state search calculations which use two possible configurations for final structures, namely with the two $\mathrm{H}$ atoms on the same side (named as $\mathrm{FS}_{1}$ ) or on both sides (named as $\mathrm{FS}_{2}$ ). In light of our DFT calculations, the $\mathrm{FS}_{1}$ structure with a slightly higher energy of $0.2 \mathrm{eV}$ is less stable than the $\mathrm{FS}_{2}$ structure. Previous studies indicate that the $\mathrm{H}$ atom introduced by proton irradiation will saturate a $\mathrm{C}$ dangling bond and produce the $\mathrm{FS}_{2}$ structure. ${ }^{17}$ However, our case shows that the reaction from the IS to $\mathrm{FS}_{2}$ is absent due to the difficulty of the $\mathrm{H}$ atom going through the vacancy to the other side of the graphene. Thus, only the reaction from the IS to $\mathrm{FS}_{1}$ is discussed in the following.

After LST/QST and NEB calculations, the detailed reaction pathway and the energy barrier for the dissociative adsorption of a $\mathrm{H}_{2}$ molecule on graphene with a mono-atom-vacancy are shown in Fig. 2b. Besides the initial and final structures, the atomic structure of the TS is also shown in Fig. 2b. It is found
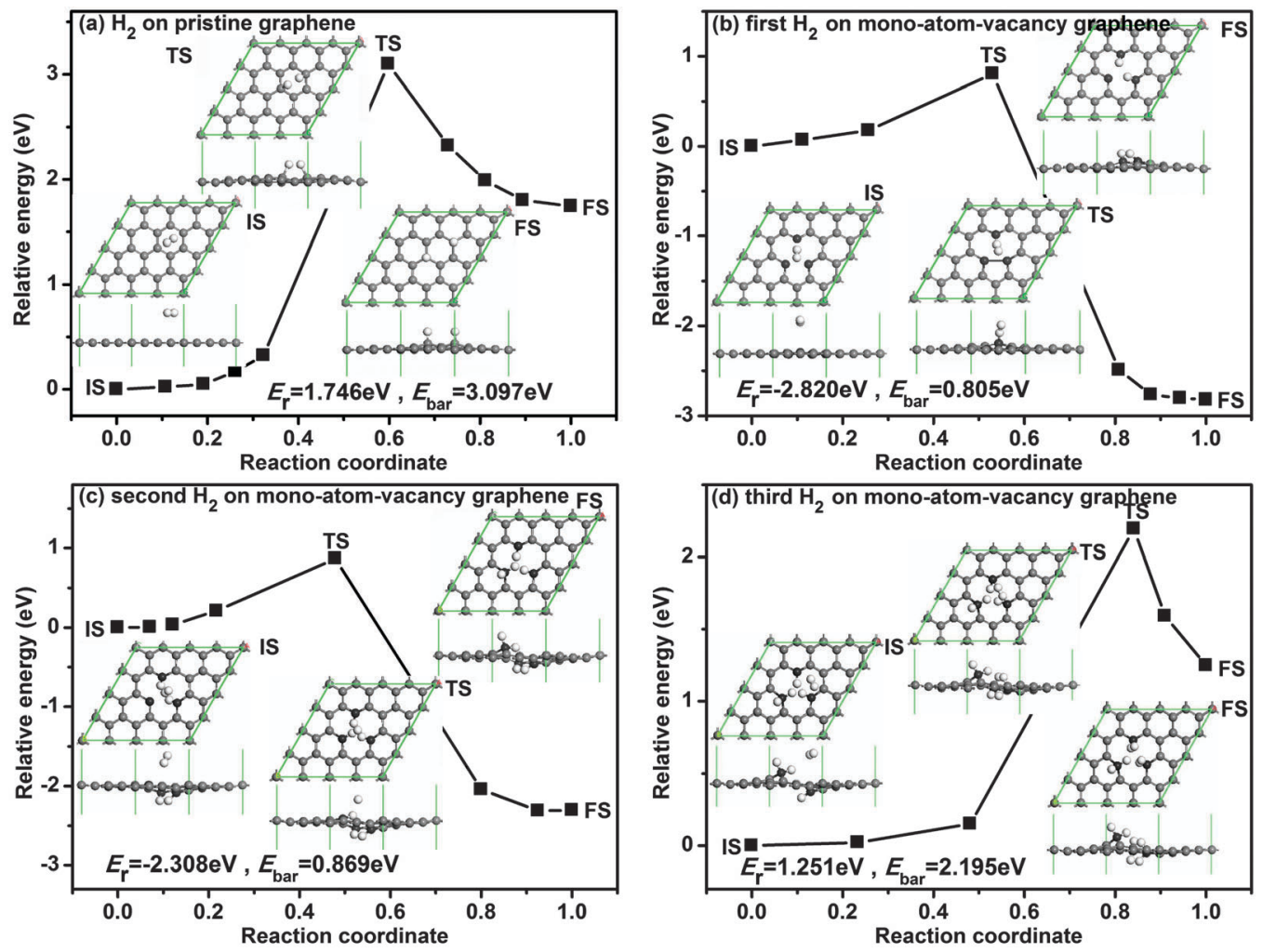

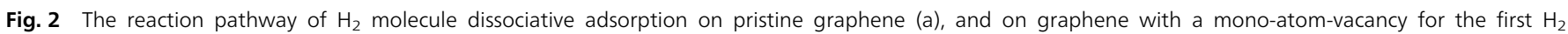

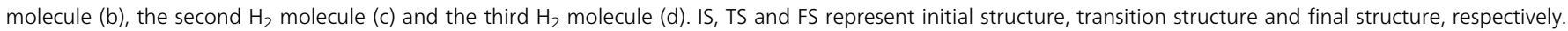

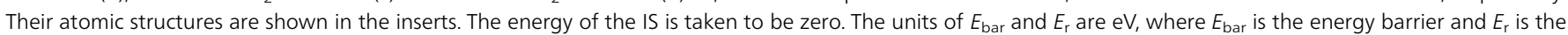
reaction energy. The grey, black and white atoms are saturated $C$, unsaturated $C$ and $H$, respectively. 
Table 1 The adsorption energy of hydrogen in the initial structure $\left(E_{\text {ad_Is }}\right)$ and final structure $\left(E_{\text {ad_Es }}\right)$, the distance of the $\mathrm{H}$ atom above the graphene $D$, the $\mathrm{H}-\mathrm{H}$ bond length $L_{H-H}$ and the Mulliken atomic charge of $\mathrm{H}_{2}$ adsorption on graphene $Q_{H_{2}}$

\begin{tabular}{|c|c|c|c|c|c|}
\hline & $E_{\text {ad_Is }}(\mathrm{eV})$ & $D(\AA)$ & $L_{\mathrm{H}-\mathrm{H}}(\AA)$ & $Q_{\mathrm{H}_{2}}(\mathrm{e})$ & $E_{\text {ad_Fs }}(\mathrm{eV})$ \\
\hline 1st $\mathrm{H}_{2}$ on mono-atom-vacancy & -0.107 & 2.791 & 0.751 & -0.002 & -2.927 \\
\hline
\end{tabular}

that the energy barrier decreases significantly on the defective graphene, $E_{\mathrm{bar}}$ decreases from $3.097 \mathrm{eV}$ in the pristine graphene system to $0.805 \mathrm{eV}$. The dissociative energy barrier for the first $\mathrm{H}_{2}$ molecule on the defective graphene is slightly smaller than the reported result of $1.1 \mathrm{eV},{ }^{18}$ probably due to the different cluster graphite models and the mixed quantum mechanics/ molecular mechanics methods used in the literature and in this work. In addition, the reaction also changes from being endothermic to exothermic with a negative reaction energy $E_{\mathrm{r}}$ of $-2.820 \mathrm{eV}$. Since a surface reaction at ambient temperature may occur when the critical barrier of $E_{\mathrm{cbar}}$ is smaller than $0.91 \mathrm{eV},{ }^{35}$ this dissociative adsorption should spontaneously occur at room temperature.

After the dissociative adsorption of the first $\mathrm{H}_{2}$ molecule, two $\sigma$ dangling bonds are saturated with $\mathrm{H}$ atoms, while one remaining dangling bond is still active, which may induce the adsorption of the second $\mathrm{H}_{2}$ molecule. For the second $\mathrm{H}_{2}$, it prefers to adsorb on the other side of the graphene opposite to the first $\mathrm{H}_{2}$. The preferred configuration with $E_{\text {ad_IS }}=0.159 \mathrm{eV}$ is shown as the IS in Fig. 2c where both dissociated $\mathrm{H}$ atoms adsorb on the same $\mathrm{C}$ atom with the dangling bond as the most stable configuration, which is taken as the FS. Note that after the second $\mathrm{H}_{2}$ adsorption, the first two $\mathrm{H}$ atoms and the two $\mathrm{C}$ atoms that bind with the first two $\mathrm{H}$ atoms, are pushed further out of the graphene layer. LST/QST and NEB calculations give $E_{\mathrm{bar}}=0.869 \mathrm{eV}$, and $E_{\mathrm{r}}=-2.308 \mathrm{eV}$. Therefore, the dissociative adsorption for the second $\mathrm{H}_{2}$ molecule can also occur smoothly at room temperature. Further dissociative adsorption of a third $\mathrm{H}_{2}$ leads not only to a larger $E_{\mathrm{bar}}=2.195 \mathrm{eV}>E_{\mathrm{cbar}}=0.91 \mathrm{eV},{ }^{35}$ but also to a positive $E_{\mathrm{r}}=1.251 \mathrm{eV}>0$ (see Fig. 2d). As a result, the reaction can not proceed at room temperature. It is noteworthy that if we increase the size of the vacancy to two $\mathrm{C}$ atoms, $E_{\text {bar }}=2.166 \mathrm{eV}$ for one $\mathrm{H}_{2}$ molecule dissociative adsorption is found, indicating that this dissociative adsorption is highly unlikely at room temperature. Therefore, only the graphene system with a mono-atom-vacancy is valid for hydrogenation.

The band structure of graphene with a mono-atom-vacancy is shown in Fig. 3b, while Fig. 3a shows that of pristine graphene for comparison purposes. As expected, the bands close at the $K$ point with the zero band gap for pristine graphene. For graphene with a
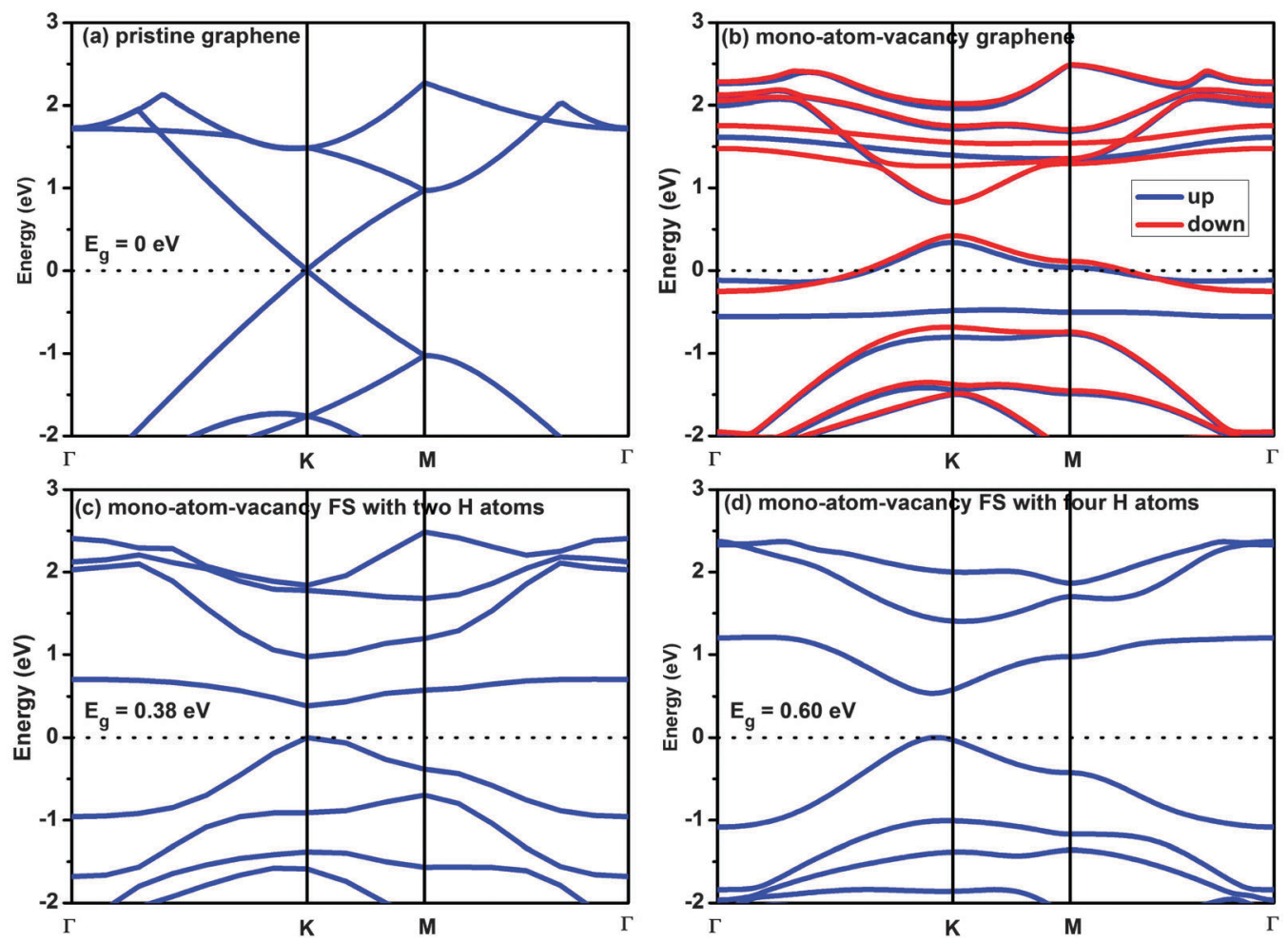

Fig. 3 The band structures of pristine graphene (a), graphene with a mono-atom-vacancy (b), the mono-atom-vacancy FS after the first dissociative adsorption of $\mathrm{H}_{2}$ (c), and the mono-atom-vacancy FS after the second dissociative adsorption $\mathrm{H}_{2}$ (d) in a $4 \times 4$ supercell. The dotted line denotes the Fermi level. 
(a) mono-atom-vancancy on $7 \times 7$ supercell
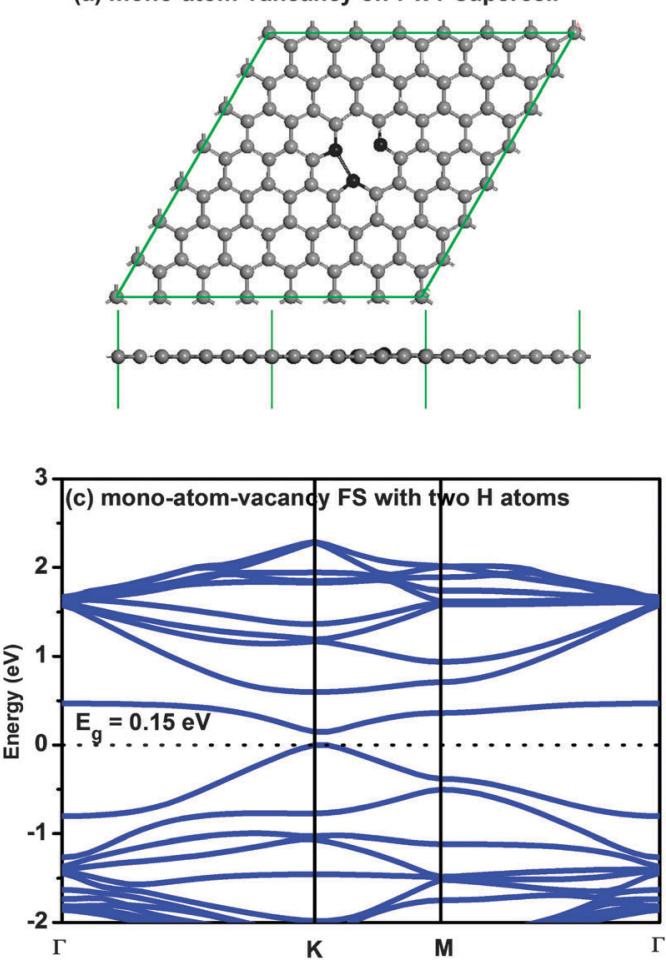
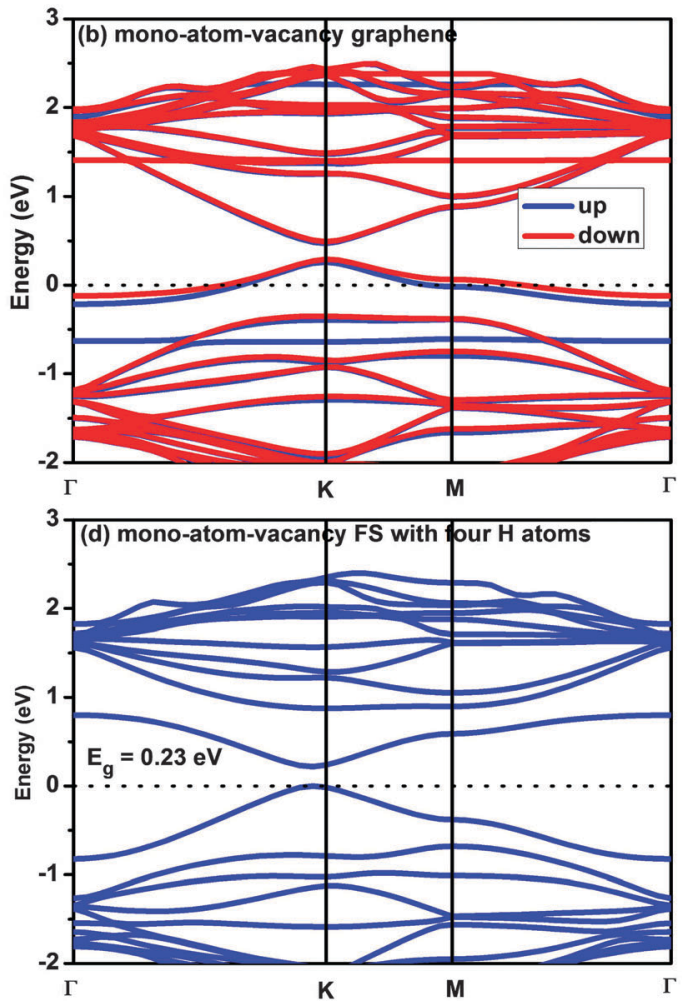

Fig. 4 The favorable configurations of graphene with a mono-atom-vacancy in a $7 \times 7$ supercell (a). The band structures of graphene with a mono-atom-vacancy (b), the mono-atom-vacancy FS after the first $\mathrm{H}_{2}$ dissociative adsorption (c), and the mono-atom-vacancy FS after the second $\mathrm{H}_{2}$ dissociative adsorption (d).

mono-atom-vacancy, the Fermi level goes down to the valence band due to the electron deficiency, indicating p-type doping. From Fig. $3 \mathrm{c}$ and d, we can see that the band across the Fermi level is mainly contributed by the half filled $\pi$ bond, while the half filled $\sigma$ bond is responsible for the spin state. The $\pi$ bond has highly mobility due to the its delocalized nature, which implies that the hole mobility should be large and the graphene with a monoatom-vacancy should be conductive. When the first $\mathrm{H}_{2}$ is dissociated into two $\mathrm{H}$ atoms and adsorbed on the same side of the graphene as the mono-atom-vacancy (see Fig. 3c), the Fermi level moves up to meet the top of the valence band exactly and the graphene changes into a pristine semiconductor with band gap of $0.38 \mathrm{eV}$. Therefore, the adsorption of two $\mathrm{H}$ atoms degrades the conductivity of the graphene. After dissociative adsorption of the second $\mathrm{H}_{2}$ (see Fig. 3d), the band gap increases slightly to $0.60 \mathrm{eV}$ and the graphene is still a pristine semiconductor. This indicates that the change in conductivity should be small and it may be difficult to detect the dissociative adsorption of the second $\mathrm{H}_{2}$. Thus, the detection of $\mathrm{H}_{2}$ molecules mainly depends on the dissociative adsorption of the first $\mathrm{H}_{2}$ molecule. Therefore, the dissociative adsorption of $\mathrm{H}_{2}$ molecules near the vacancy can eliminate the spin state and induce a significant conductivity change, which is desirable for applications in hydrogen sensors. Although the dissociative adsorption of the second and the third $\mathrm{H}_{2}$ molecules are studied in order to comprehensively understand the reaction between the $\mathrm{H}_{2}$ molecule and the defective graphene, these dissociative adsorptions have little effect on the conductivity.
In reality, graphene may have a smaller vacancy concentration locally due to its disorder distribution. In order to investigate the effect of vacancy concentration on the electronic properties of graphene before and after $\mathrm{H}_{2}$ dissociative adsorption, we further investigated the electronic structure in $7 \times 7$ supercell with a smaller vacancy concentration of $1 / 98$ as shown in Fig. 4a. Fig. $4 \mathrm{~b}-\mathrm{d}$ show the band structures of graphene, graphene after the first $\mathrm{H}_{2}$ dissociative adsorption, and graphene after the second $\mathrm{H}_{2}$ dissociative adsorption, respectively. Similar changes in the electronic properties are found in this graphene system where the vacancy concentration is low. Thus, the dissociative adsorption of $\mathrm{H}_{2}$ near a vacancy can still change the graphene from p-type doping to a pristine semiconductor, which indicates that $\mathrm{H}_{2}$ molecules can still be detected even when the defect concentration is decreased.

To determine the sensitivity of graphene with a mono-atomvacancy for $\mathrm{H}_{2}$ gas detection, we proposed a phase diagram to determine the effects of $T$ and $P$ on the dissociative adsorption of hydrogen on graphene, which is calculated in light of eqn 6 and is shown in Fig. 5. It is known that the reaction occurs when $\Delta G(T, P)<0$. The figure shows that at a high $T$ or a low $P$, free $\mathrm{H}_{2}$ molecules cannot dissociatively adsorb on the monoatom-vacancy graphene. At room temperature, the first $\mathrm{H}_{2}$ molecule starts to dissociate on the graphene at a very low partial pressure ratio $\ln P_{\mathrm{H}_{2}} / P^{0}$ of $10^{-76}$, or $10^{-35} \mathrm{~mol} \mathrm{~L}^{-1}$. When partial pressure ratio $\ln P_{\mathrm{H}_{2}} / P^{0}>10^{-59}$, i.e. $10^{-27} \mathrm{~mol} \mathrm{~L}^{-1}$, the second $\mathrm{H}_{2}$ molecule begins to dissociate and is adsorbed on graphene. To better understand the effects of $T$ and $P$ on the 


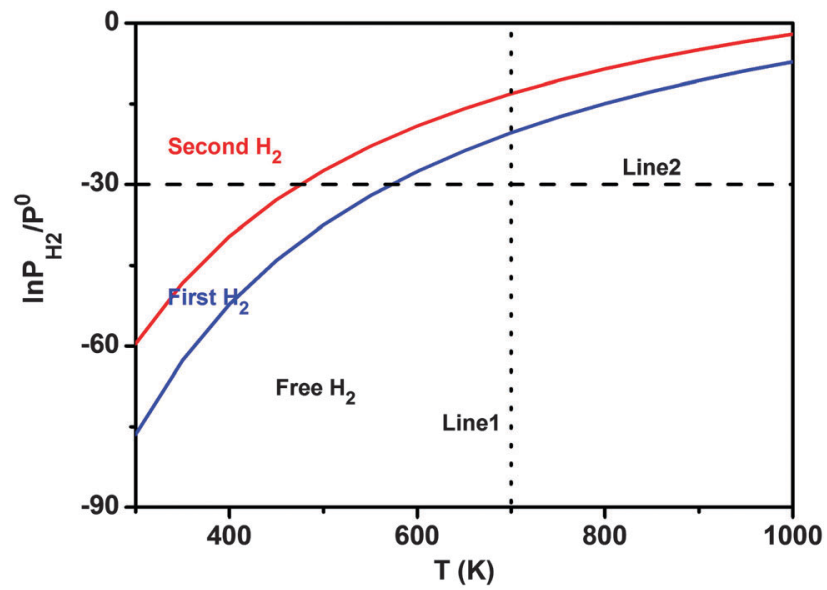

Fig. 5 The phase diagram under different temperatures $T$ and partial pressures $P / P_{0}$ for the first dissociative adsorption of a $\mathrm{H}_{2}$ molecule on mono-atom-vacancy graphene (a), and that for the second $\mathrm{H}_{2}$ molecule (b). Vertical line 1 denotes $T=700 \mathrm{~K}$, and Horizontal line 2 denotes $\ln P / P_{0}=-30$.

dissociative adsorption of $\mathrm{H}_{2}$ on mono-vacancy graphene, lines 1 and 2 are plotted in the figure. Line 1 shows that increasing $P$ at constant $T$ promotes dissociative adsorption. Under a certain $P$ as shown by line 2, low $T$ induces dissociative adsorption. It is reported that the frequently used $\mathrm{SnO}_{2}$ and $\mathrm{TiO}_{2}$ based sensors can detect hydrogen molecules at the low concentration range of 5-10 ppm, ${ }^{5,6}$ i.e., about $10^{-7} \mathrm{~mol} \mathrm{~L}^{-1}$; and Pd nonoparticle decorated $\mathrm{ZnO}$ nanorods can detect hydrogen molecules at the even lower concentration of $0.2 \mathrm{ppm}^{7}$ i.e., about $10^{-8} \mathrm{~mol} \mathrm{~L}^{-1}$. Compared with these results, graphene with mono-atom vacancies has ultra-high sensitivity for $\mathrm{H}_{2}$ detection at room temperature.

\section{Conclusion}

In summary, the effects of vacancies on the dissociative adsorption of $\mathrm{H}_{2}$ molecules on graphene are investigated through DFT calculations. The results show that graphene with a monoatom-vacancy can efficiently reduce the dissociative energy barrier of $\mathrm{H}_{2}$ molecules on graphene and two $\mathrm{H}_{2}$ molecules can be dissociatively adsorbed at each vacancy even when the concentration of $\mathrm{H}_{2}$ is as low as $10^{-35} \mathrm{~mol} \mathrm{~L}^{-1}$. Based on the calculated band structures, the electronic properties change significantly after the hydrogen dissociative adsorption. Therefore, graphene with a mono-atom-vacancy has potential for ultra-low concentration hydrogen detection.

\section{Acknowledgements}

We acknowledge support from National Key Basic Research, Development Program (Grant No. 2010CB631001) and China Scholarship Council (No. 201206170088). ZA acknowledges financial support from the Chancellor's Postdoctoral Research Fellowship Program of the University of Technology, Sydney, and the Goldstar Award from the University of New South Wales (RG124422). SL would also like to thank the financial support from Australia Research Council (FT FT100100956).

\section{References}

1 M. Balat, Int. J. Hydrogen Energy, 2008, 33, 4013-4029.

2 W. J. Buttner, M. B. Post, R. Burgess and C. Rivkin, Int. J. Hydrogen Energy, 2011, 36, 2462-2470.

3 O. K. Varghese, D. Gong, M. Paulose, K. G. Ong and C. A. Grimes, Sens. Actuators, B, 2003, 93, 338-344.

4 G. K. Mor, O. K. Varghese, M. Paulose, K. G. Ong and C. A. Grimes, Thin Solid Films, 2006, 496, 42-48.

5 B. Wang, L. F. Zhu, Y. H. Yang, N. S. Xu and G. W. Yang, J. Phys. Chem. C, 2008, 112, 6643-6647.

6 C. Lu and Z. Chen, Sens. Actuators, B, 2009, 140, 109-115.

7 T.-R. Rashid, D.-T. Phan and G.-S. Chung, Sens. Actuators, B, 2013, 185, 777-784.

8 T. Waitz, T. Wagner, T. Sauerwald, C.-D. Kohl and M. Tiemann, Adv. Funct. Mater., 2009, 19, 653-661.

9 K. S. Novoselov, A. K. Geim, S. V. Morozov, D. Jiang, Y. Zhang, S. V. Dubonos, I. V. Grigorieva and A. A. Firsov, Science, 2004, 306, 666-669.

10 K. S. Novoselov, A. K. Geim, S. V. Morozov, D. Jiang, M. I. Katsnelson, I. V. Grigorieva, S. V. Dubonos and A. A. Firsov, Nature, 2005, 438, 197-200.

11 C. G. Lee, X. D. Wei, J. W. Kysar and J. Hone, Nature, 2008, 321, 385-388.

12 Y. Miura, H. Kasai, W. Diño, H. Nakanishi and T. Sugimoto, J. Appl. Phys., 2003, 93, 3395-3400.

13 F. Banhart, J. Kotakoski and A. V. Krasheninnikov, ACS Nano, 2011, 5, 26-41.

14 D. Li, M. B. Muller, S. Gilje, R. B. Kaner and G. G. Wallace, Nat. Nanotechnol., 2008, 3, 101-105.

15 L. Tapasztó, G. Dobrik, P. Nemes-Inczel, G. Vertesy, P. Lambin and L. P. Biró, Phys. Rev. B: Condens. Matter Mater. Phys., 2008, 78, 233407.

16 O. Lehtinen, J. Kotakoski, A. V. Krasheninnikov, A. Tolvanen, K. Nordlund and J. Keinonen, Phys. Rev. B: Condens. Matter Mater. Phys., 2010, 81, 153401.

17 P. O. Lehtinen, A. S. Foster, Y. C. Ma, A. V. Krasheninnikov and R. M. Nieminen, Phys. Rev. Lett., 2004, 93, 187202.

18 A. Allouche and Y. Ferro, Carbon, 2006, 44, 3320-3327.

19 B. H. Kim, S. J. Hong, S. J. Baek, H. Y. Jeong, N. Park, M. Lee, S. W. Lee, M. Park, S. W. Chu, H. S. Shin, J. Lim, J. C. Lee, Y. Jun and Y. W. Park, Sci. Rep., 2012, 2, 690.

20 B. Delley, J. Chem. Phys., 2000, 113, 7756-7764.

21 J. P. Perdew, K. Burke and M. Ernzerhof, Phys. Rev. Lett., 1996, 77, 3865.

22 A. Roldán, J. M. Ricart and F. Illas, Theor. Chem. Acc., 2009, 123, 119-126.

23 T. A. Halgren and W. N. Lipscomb, Chem. Phys. Lett., 1977, 49, 225-232.

24 G. Henkelman and H. Jonsson, J. Chem. Phys., 2000, 113, 9978-9985.

25 S. Grimme, J. Comput. Chem., 2006, 27, 1787-1789.

26 K. Reuter and M. Scheffler, Phys. Rev. B: Condens. Matter, 2001, 65, 035406.

27 Z. X. Yang, Q. G. Wang, S. Y. Wei, D. W. Ma and Q. Sun, J. Phys. Chem. C, 2010, 114, 14891-14899. 
28 M. D. Segall, P. L. D. Lindan, M. J. Probert, C. J. Pickard, P. J. Hasnip, S. J. Clark and M. C. Payne, J. Phys.: Condens. Matter, 2002, 14, 2717-2744.

29 K. Reuter and M. Scheffler, Phys. Rev. B: Condens. Matter, 2001, 65, 035406.

30 NIST Chemistry Web book: http://webbook.nist.gov/.

31 C. Q. Sun, Y. G. Nie, J. S. Pan, X. Zhang, S. Z. Ma, Y. Wang and W. T. Zheng, RSC Adv., 2012, 2, 2377-2383.
32 M. Amft, S. Lebègue, O. Eriksson and N. V. Skorodumova, J. Phys.: Condens. Matter, 2011, 23, 395001.

33 E. Ganz, K. Sattler and J. Clarke, Surf. Sci., 1989, 219, 33-67. 34 F. Costanzo, P. L. Silvestrelli and F. Ancilotto, J. Chem. Theory Comput., 2012, 8, 1288-1294.

35 D. C. Young, Computational Chemistry: A Practical Guide for Applying Techniques to Real World Problems, Wiley, New York, 2001. 